HETEROCYCLES, Vol. 96, No. 10, 2018, pp. 1821 - 1827. @ 2018 The Japan Institute of Heterocyclic Chemistry Received, 5th September, 2018, Accepted, 26th September, 2018, Published online, 19th October, 2018 DOI: $10.3987 / C O M-18-13983$

\title{
ONE-POT SYNTHESIS OF 6H-INDOLO[2,3-b]QUINOLINES FROM 2-NITROBENZALDEHYDE AND INDOLE DERIVATIVES VIA DOMINO REACTION
}

\section{Wei-Nan Zhang, ${ }^{1}$ Jiao Xu, ${ }^{2 *}$ Dao-Lin Wang, ${ }^{1 *}$ and Zhen-Kai Zhong ${ }^{1}$}

${ }^{1}$ College of Chemistry and Chemical Engineering, Bohai University, Jinzhou, China; ${ }^{2}$ College of Pharmacy, Heilongjiang University of Chinese Medicine, Harbin, China. *Corresponding authors E-mail: xujiao2007@sina.com; wangdaolin@sina.com

\begin{abstract}
An efficient and simple method for the synthesis of $6 H$-indolo[2,3-b]quinolones was described. The construction of this fused heterocycles system was achieved by alkylation-reduction-cyclization domino reaction of 2-nitrobenzaldehyde with indoles using $\mathrm{SnCl}_{2}$ as a promoter in $[\mathrm{bmim}] \mathrm{Br}$ medium.
\end{abstract}

The indole-fused heterocyclic system is frequently found in natural products isolated from plants used in traditional medicine. Due to the broad spectrum of biological and pharmacological activities associated with compounds incorporating this structural skeleton, it has been recognized as an important privileged scaffold in drug discovery. ${ }^{1}$ Particularly, indoloquinolines have been well established to be useful as antibacterial, antifungal, antimalarial, anticancer, antiplatelet, antihypertensive agents as well as exhibiting several other activities. ${ }^{2}$ It is believed that they act as DNA intercalating agents ${ }^{3}$ and topoisomerase II inhibitors. ${ }^{4}$ Among them, $6 H$-indolo[2,3-b]quinoline is the precursor to cryptotakieine (neocryptolepine) alkaloid, ${ }^{5}$ and displays a strong antiplasmodial activity ${ }^{6}$ in addition to antimicrobial and cytotoxic activity. ${ }^{7}$ So development of a new and efficient methodology for the synthesis of biologically potent indolo[2,3-b]quinolines has drawn great attention of synthetic as well as medicinal chemists. ${ }^{8}$ $\mathrm{SnCl}_{2} \cdot 2 \mathrm{H}_{2} \mathrm{O}$ is an effective reagent that has been used for various reductive cyclization processes due to its easy availability, environmental safety, low cost and water-tolerant catalyst. ${ }^{9}$ In 2008 , Kundu and co-workers reported the synthesis of $6 H$-indolo[2,3-b] quinoline derivatives from 2-substituted nitroarenes under refluxing conditions in methanol by using $\mathrm{SnCl}_{2} \cdot 2 \mathrm{H}_{2} \mathrm{O} .{ }^{8 \mathrm{~d}}$ However, in this method, the preparation of starting materials involved multiple steps.

Most of the known procedures are associated with several shortcomings with respective to the formation 
of side products, use of toxic solvents, tedious procedures of work-up and use of expensive catalysts. Hence, the development of a simple, convenient and environmentally benign method for the synthesis of $6 H$-indolo[2,3-b]quinoline derivatives is still a welcoming topic in organic synthesis.

On the other hand, domino reactions have emerged as an effective tool for the assembly of complex cyclic structures by the combination of two or more distinct reactions into a one-pot transformation. ${ }^{10}$ Recently, we have developed several domino reactions toward the fused heterocycles system. ${ }^{11}$ In continuation of this project, we now report the $6 H$-indolo[2,3-b]quinolines 3 preparation via domino reaction from 2-nitrobenzaldehyde $\mathbf{1}$ with indoles $\mathbf{2}$, as a stable and inexpensive starting material, providing indole-fused quinolines by $\mathrm{SnCl}_{2} \cdot 2 \mathrm{H}_{2} \mathrm{O}$ as a promoter in [bmim] Br medium (Scheme 1).<smiles>[R]c1ccc([N+](=O)[O-])c(C=O)c1</smiles>

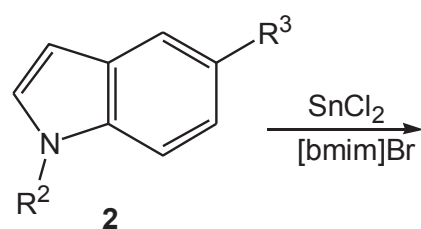

2

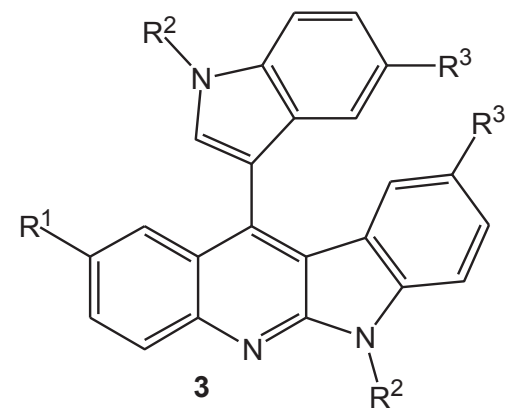

3

Scheme 1. Synthesis of $6 H$-indolo[2,3-b]quinoline derivatives

In our initial study, various reaction conditions including solvents and temperatures were tested in the synthesis of 11-(1H-indol-3-yl)-6H-indolo[2,3-b]quinoline 3a from indole 1a and 2-nitro- benzaldehyde 2a using $\mathrm{SnCl}_{2} \cdot 2 \mathrm{H}_{2} \mathrm{O}$ as a promoter. Different solvents, such as $\mathrm{EtOH}, \mathrm{MeCN}, \mathrm{AcOH}$, and ionic liquids $\left([\mathrm{bmim}] \mathrm{Br},[\mathrm{bmim}] \mathrm{BF}_{4}\right)$ were examined, respectively. The results are summarized in Table 1 . The best result was obtained when the reaction was carried out in $[\mathrm{bmim}] \mathrm{Br}$ at $80{ }^{\circ} \mathrm{C}$ in $76 \%$ yield.

When the same reactions were performed at room temperature for $2 \mathrm{~h}$, only a very trace amount of product 3a was formed, the corresponding bis(indolyl)methane was isolated in $85 \%$. In the experimental procedure, indole and aldehydes underwent smooth transformation (3-6 h) to the corresponding bis(indolyl)methanes in ionic liquid in good yields. ${ }^{12}$ However, in the absence of ionic liquid, the reaction did not yield any product even after a long reaction period (10-15 h).

In addition, heating the solution of $\mathbf{1 a}$ and $\mathbf{2 a}$ in an acetic acid and concentrated hydrochloric acid $(\mathrm{v} / \mathrm{v}, 1: 2)$ at reflux for $12 \mathrm{~h}$ using commonly used zinc dust as the reductant led to an unexpected conjugated tetracyclic product 3a in 52\% yield. Iron powder was less active for this transformation (20\%). 
Table 1. Optimization of reaction conditions on the synthesis of $\mathbf{3 a}$ *

\begin{tabular}{|c|c|c|c|c|c|}
\hline Entry & Catalyst / (eq.) & Solvent & Temp $\left({ }^{\circ} \mathrm{C}\right)$ & Time (h) & Yield (\%) \\
\hline 1 & $\mathrm{SnCl}_{2} \cdot 2 \mathrm{H}_{2} \mathrm{O}(1.0)$ & $\mathrm{EtOH}$ & 80 & 24 & 43 \\
\hline 2 & $\mathrm{SnCl}_{2} \cdot 2 \mathrm{H}_{2} \mathrm{O}(1.0)$ & $\mathrm{MeCN}$ & 80 & 24 & 36 \\
\hline 3 & $\mathrm{SnCl}_{2} \cdot 2 \mathrm{H}_{2} \mathrm{O}(1.0)$ & $\mathrm{AcOH}$ & 120 & 18 & 61 \\
\hline 4 & $\mathrm{SnCl}_{2} \cdot 2 \mathrm{H}_{2} \mathrm{O}(1.0)$ & [bmim] Br & 80 & 4 & 76 \\
\hline 5 & $\mathrm{SnCl}_{2} \cdot 2 \mathrm{H}_{2} \mathrm{O}(1.0)$ & [bmim] Br & 100 & 4 & 75 \\
\hline 6 & $\mathrm{SnCl}_{2} \cdot 2 \mathrm{H}_{2} \mathrm{O}(1.0)$ & [bmim] Br & 120 & 2 & 63 \\
\hline 7 & $\mathrm{SnCl}_{2} \cdot 2 \mathrm{H}_{2} \mathrm{O}(1.0)$ & {$[\mathrm{bmim}] \mathrm{BF}_{4}$} & 80 & 5 & 70 \\
\hline 8 & $\mathrm{SnCl}_{2} \cdot 2 \mathrm{H}_{2} \mathrm{O}(0.8)$ & [bmim] Br & 80 & 8 & 72 \\
\hline 9 & $\mathrm{SnCl}_{2} \cdot 2 \mathrm{H}_{2} \mathrm{O}(1.2)$ & [bmim] Br & 80 & 4 & 73 \\
\hline 10 & $\mathrm{Zn}-\mathrm{HCl}(1.5)$ & HOAc & 80 & 12 & 52 \\
\hline 11 & $\mathrm{Zn}-\mathrm{HCl}(1.5)$ & [bmim] Br & 80 & 10 & 55 \\
\hline 12 & $\mathrm{Fe}-\mathrm{HCl}(1.5)$ & HOAc & 80 & 10 & 20 \\
\hline
\end{tabular}

*Reaction conditions: indole (1, $2.0 \mathrm{mmol})$, 2-nitrobenzaldehyde (2a, $1.0 \mathrm{mmol})$, solvent (5 mL).

Under these optimized reaction conditions, a series of $6 H$-indolo[2,3-b]quinoline derivatives 3 were synthesized. As shown in Table 2, the reaction was successful for 2-nitrobenzaldehyde $\mathbf{1}$ and indoles 2 incorporating $\mathrm{R}$ groups carrying either electron-donating or electron-withdrawing substituents reacted efficiently giving good yields (70-83\%).

Table 2. Synthesis of $6 H$-indolo[2,3- $b]$ quinolines 3

\begin{tabular}{|c|c|c|c|c|c|}
\hline Entry & $\mathrm{R}^{1}(\mathbf{1})$ & $\mathbf{R}^{2} / \mathrm{R}^{3}(\mathbf{2})$ & Time (h) & Product (3) & Yield (\%) \\
\hline 1 & 1a $\mathrm{H}$ & 2a $\mathrm{H} / \mathrm{H}$ & 4 & $3 \mathbf{a}$ & 76 \\
\hline 2 & 1b $\mathrm{Cl}$ & 2a $\mathrm{H} / \mathrm{H}$ & 5 & $3 b$ & 70 \\
\hline 3 & 1a $\mathrm{H}$ & 2b $\mathrm{Me} / \mathrm{H}$ & 4 & $3 c$ & 80 \\
\hline 4 & 1b $\mathrm{Cl}$ & 2b $\mathrm{Me} / \mathrm{H}$ & 5 & $3 d$ & 83 \\
\hline 5 & 1a $\mathrm{H}$ & 2c $\mathrm{H} / \mathrm{Br}$ & 7 & $3 \mathbf{e}$ & 76 \\
\hline 6 & 1b $\mathrm{Cl}$ & $\mathbf{2 d} \quad \mathrm{H} / \mathrm{OMe}$ & 5 & $3 f$ & 78 \\
\hline 7 & 1a $\mathrm{H}$ & $\mathrm{H} / \mathrm{CN}$ & 6 & $3 g$ & 75 \\
\hline 8 & 1b $\mathrm{Cl}$ & 2f $\mathrm{H} / \mathrm{Br}$ & 7 & $3 h$ & 74 \\
\hline
\end{tabular}


The proposed mechanism of the process is summarized in Scheme 2. Firstly, 2-nitrobenzaldehyde 1 reacts with indole 2 to generate bis(indolyl)methane $\mathbf{A}$ via alkylation of indole in the presence of $\mathrm{SnCl}_{2}$ as cartalyst. ${ }^{12,13}$ Meanwhile, intermediate $\mathbf{B}$ could be obtained via a reduction of the nitro gruop by $\mathrm{SnCl}_{2}$. Subsequent prototropy to $\mathbf{C}$ initiates a $6 \pi$ electrocyclization to $\mathbf{D}$ from which the products 3 result following dehydration (Scheme 2).
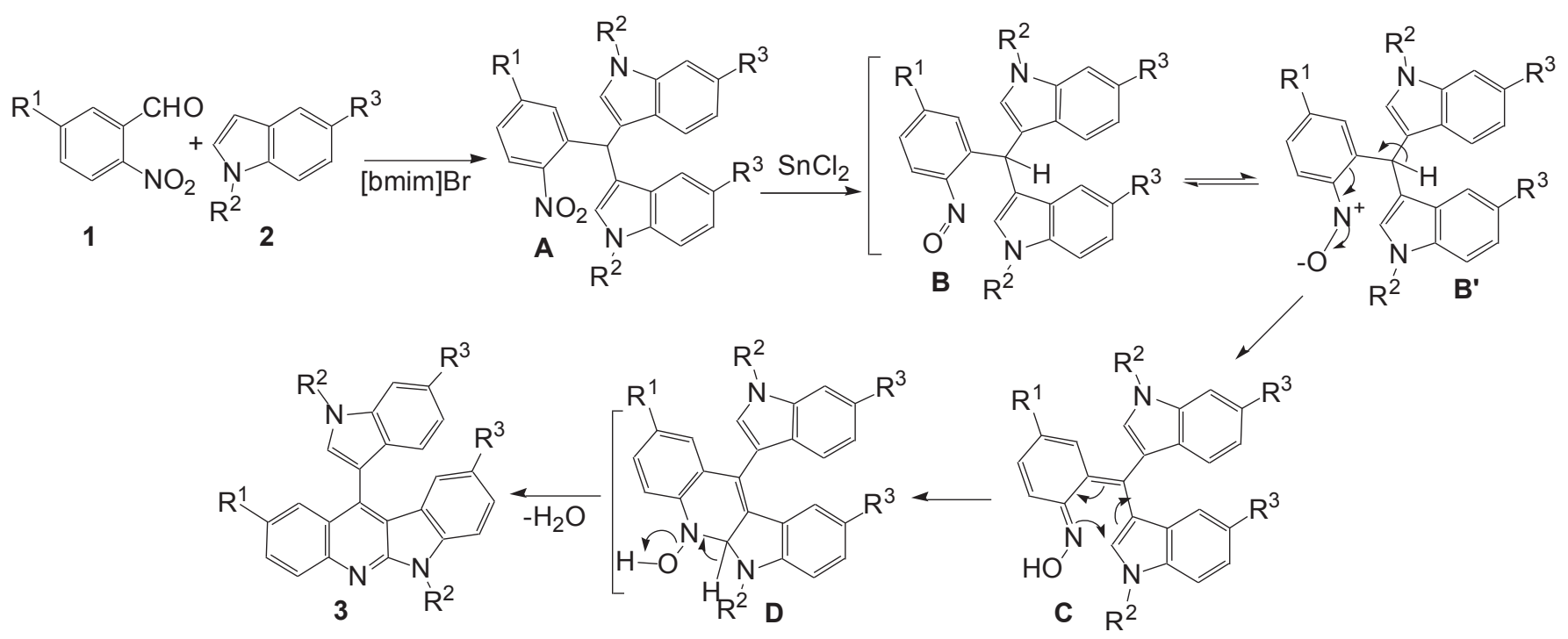

Scheme 2. Proposed mechanism for the synthesis of indolo[2,3-b]quinolines 3

In summary, we have demonstrated the efficient method for the synthesis of $6 H$-indolo[2,3- $b]$ quinolines from 2-nitrobenzaldehyde and indoles through alkylation-reduction-cyclization domono reaction. This approach offers an effective route for the construction of indolo[2,3-b]quinoline frameworks in a one-step process from commercially available starting materials.

\section{EXPERIMENTAL}

Melting points were determined in open capillaries and are uncorrected. The NMR spectra were recorded with a Bruker Avance 400 spectrometer (400 MHz for ${ }^{1} \mathrm{H}$ and $100 \mathrm{MHz}$ for ${ }^{13} \mathrm{C}$ ) using TMS an internal reference. IR spectra were measured on Shimadzu FTIR-8300 spectrophotometer. C, H and N analyses were performed by a HP-MOD 1106 microanalyzer.

Typical Procedure for the Preparation of 11-(1H-Indol-3-yl)-6H-indolo[2,3-b]quinolines. To a solution of 2-nitrobenzaldehydes $(\mathbf{1}, 1.0 \mathrm{mmol})$ and indoles $(\mathbf{2}, 2.0 \mathrm{mmol})$ in $[\mathrm{bmim}] \mathrm{Br}(5.0 \mathrm{~mL})$ $\mathrm{SnCl}_{2} \cdot 2 \mathrm{H}_{2} \mathrm{O}(1.0 \mathrm{mmol})$ was added, and the reaction was heated at $80{ }^{\circ} \mathrm{C}$. After completion monitored by TLC, the reaction mixture was allowed to cool to room temperature, and then water $(20 \mathrm{~mL})$ was added to the mixture. EtOAc $(50 \mathrm{~mL})$ was added to the mixture. The organic layer was washed with brine $(50 \mathrm{~mL})$, dried over anhydrous $\mathrm{Na}_{2} \mathrm{SO}_{4}$ and evaporated to dryness. The residue was recrystallized from isopropanol to afford the corresponding products $\mathbf{3 a}-\mathbf{h}$. 
11-(1H-Indol-3-yl)-6H-indolo[2,3-b]quinoline (3a): ${ }^{8 \mathrm{~h}}$ Yellow crystals. mp 260-262 ${ }^{\circ} \mathrm{C}$; IR (KBr): $v$ 3325, 3052 1602, $1590 \mathrm{~cm}^{-1} ;{ }^{1} \mathrm{HNMR}\left(400 \mathrm{MHz}, \mathrm{CDCl}_{3}\right): \delta 7.21$ (dd, $\left.J=6.8,7.2 \mathrm{~Hz}, 1 \mathrm{H}\right), 7.42(\mathrm{~d}, J=$ $8.8 \mathrm{~Hz}, 1 \mathrm{H}), 7.61-7.69(\mathrm{~m}, 2 \mathrm{H}), 7.74(\mathrm{dd}, J=8.0,8.2 \mathrm{~Hz}, 1 \mathrm{H}), 7.77-7.82(\mathrm{~m}, 4 \mathrm{H}), 8.04-8.05(\mathrm{~m}, 1 \mathrm{H})$, $8.11(\mathrm{~d}, J=8.4 \mathrm{~Hz}, 1 \mathrm{H}), 8.42$ (d, $J=8.4 \mathrm{~Hz}, 1 \mathrm{H}), 8.54(\mathrm{~d}, J=7.6 \mathrm{~Hz}, 1 \mathrm{H}), 11.1(\mathrm{~s}, 1 \mathrm{H}), 12.0(\mathrm{~s}, 1 \mathrm{H}) .{ }^{13} \mathrm{C}$ NMR (100 MHz, $\left.\mathrm{CDCl}_{3}\right): \delta 107.8,112.3,112.5,119.7,119.9,120.0,120.5,121.7,121.8,122.2,124.9$, $125.9,126.1,126.2,127.1,127.3,129.6,129.8,132.0,137.0,144.4,144.6,145.6$. Anal. Calcd for $\mathrm{C}_{23} \mathrm{H}_{15} \mathrm{~N}_{3}$ : C 82.86, H 4.54, N 12.60. Found: C 82.91, H 4.57, N 12.64.

2-Chloro-11-(1H-indol-3-yl)-6H-indolo[2,3-b]quinoline (3b): ${ }^{8 \mathrm{~h}}$ Yellow crystals. $\mathrm{mp}>300{ }^{\circ} \mathrm{C}$; IR (KBr): v 3341, 3024, 1607, $1595 \mathrm{~cm}^{-1} ;{ }^{1} \mathrm{H}$ NMR (400 MHz, $\left.\mathrm{CDCl}_{3}\right): \delta$ 7.04-7.05 (m, 1H), 7.17-7.26 (m, $3 \mathrm{H}), 7.56-7.65(\mathrm{~m}, 4 \mathrm{H}), 7.93-7.96(\mathrm{~m}, 2 \mathrm{H}), 8.27(\mathrm{~d}, J=8.4 \mathrm{~Hz}, 1 \mathrm{H}), 8.38(\mathrm{~d}, J=7.6 \mathrm{~Hz}, 1 \mathrm{H}), 11.1(\mathrm{~s}, 1 \mathrm{H})$, 11.9 (s, 1H). ${ }^{13} \mathrm{C}$ NMR (100 MHz, $\left.\mathrm{CDCl}_{3}\right): \delta 107.2,112.4,112.7,119.7,119.8,120.0,120.3,121.7$, $121.9,122.3,124.4,126.6,126.9,127.1,127.4,129.5,130.2,131.7,132.5,137.0,142.7,144.8,146.2$. Anal. Calcd for $\mathrm{C}_{23} \mathrm{H}_{14} \mathrm{ClN}_{3}$ : C 75.10, H 3.84, N 11.42. Found: C 75.17, H 3.86, N 11.46.

6-Methyl-11-(1-methy-1H-lindol-3-yl)-6H-indolo[2,3-b]quinoline (3c): ${ }^{8 a}$ Yellow crystals. mp 261$263{ }^{\circ} \mathrm{C}$; IR (KBr): v 3031, 1613, $1597 \mathrm{~cm}^{-1} ;{ }^{1} \mathrm{H}$ NMR (400 MHz, $\left.\mathrm{CDCl}_{3}\right): \delta 3.44(\mathrm{~s}, 3 \mathrm{H}), 4.16(\mathrm{~s}, 3 \mathrm{H})$, $7.43(\mathrm{dd}, J=7.6,8.0 \mathrm{~Hz}, 1 \mathrm{H}), 7.49$ (dd, $J=7.4,8.0 \mathrm{~Hz}, 1 \mathrm{H}), 7.57$ (dd, $J=7.6,8.0 \mathrm{~Hz}, 1 \mathrm{H}), 7.69-7.72$ (m, 3H), 7.83-7.88 (m, 3H), 7.89-7.92 (m, 2H), 8.42 (d, $J=7.6 \mathrm{~Hz}, 1 \mathrm{H}), 8.59$ (d, $J=7.6 \mathrm{~Hz}, 1 \mathrm{H}) .{ }^{13} \mathrm{C}$ NMR $\left(100 \mathrm{MHz}, \mathrm{CDCl}_{3}\right): \delta 31.4,33.3,106.9,110.0,110.8,119.5,119.8,120.2,120.3,121.3,121.6,122.3$, 125.4, 125.6, 126.5, 128.0, 129.4, 129.6, 130.3, 131.0, 132.6, 136.7, 143.8, 145.7, 145.9. Anal. Calcd for $\mathrm{C}_{25} \mathrm{H}_{19} \mathrm{~N}_{3}$ : C 83.08, H 5.30, N 11.63. Found: C 83.16, H 5.37, N 11.68.

2-Chloro-6-methyl-11-(1-methyl-1H-indol-3-yl)-6H-indolo[2,3-b]quinoline (3d): Yellow crystals. mp $>300{ }^{\circ} \mathrm{C}$; IR (KBr): v 3061, 1606, $1591 \mathrm{~cm}^{-1} ;{ }^{1} \mathrm{H}$ NMR (400 MHz, $\left.\mathrm{CDCl}_{3}\right): \delta 3.23$ (s, 3H), 3.98 (s, 3H), 6.99-7.05 (m, 2H), 7.26 (d, $J=7.2 \mathrm{~Hz}, 1 \mathrm{H}), 7.32$ (d, $J=7.2 \mathrm{~Hz}, 1 \mathrm{H}), 7.52$ (d, $J=8.0 \mathrm{~Hz}, 1 \mathrm{H}), 7.58-7.66$ $(\mathrm{m}, 4 \mathrm{H}), 7,74(\mathrm{~s}, 1 \mathrm{H}), 8.22(\mathrm{~d}, J=8.4 \mathrm{~Hz}, 1 \mathrm{H}), 8.38$ (d, $J=6.8 \mathrm{~Hz}, 1 \mathrm{H}) .{ }^{13} \mathrm{C} \mathrm{NMR}\left(100 \mathrm{MHz}, \mathrm{CDCl}_{3}\right): \delta$ $31.3,33.3,106.2,110.2,110.9,119.4,120.5,120.5,121.1,121.8,122.5,123.9,125.5,125.7,126.9,128.8$, 129.4, 130.0, 130.6, 131.2, 131.5, 136.7, 142.1, 146.1, 146.2. Anal. Calcd for $\mathrm{C}_{25} \mathrm{H}_{18} \mathrm{ClN}_{3}$ : C 75.85, $\mathrm{H}$ 4.58, N 10.61. Found: C 75.92, H 4.61, N 10.64.

9-Bromo-11-(5-bromo-1H-indol-3-yl)-6H-indolo[2,3-b]quinoline (3e): Yellow crystals. mp $>300{ }^{\circ} \mathrm{C}$; IR (KBr): v 3394, 3026, 1602, $1584 \mathrm{~cm}^{-1} ;{ }^{1} \mathrm{H} \mathrm{NMR}$ (400 MHz, $\left.\mathrm{CDCl}_{3}\right): \delta 7.24$ (s, 1H), 7.30 (d, J=8.4 Hz, 1H), 7.48-7.49 (m, 2H), 7.64-7.66 (m, 4H), 7.95 (s, 1H), 8.25 (d, J=8.4 Hz, 1H), 8.47 (s, 1H), 11.1 (s, 1H), $12.0(\mathrm{~s}, 1 \mathrm{H}) .{ }^{13} \mathrm{C} \mathrm{NMR}\left(100 \mathrm{MHz}, \mathrm{CDCl}_{3}\right): \delta 107.2,111.7,112.7,114.3,114.6,120.3,121.8,123.7$, $123.9,124.8,125.5,125.7,126.3,126.7,128.8,129.0,129.7,132.2,132.4,135.7,143.2,144.3,144.5$. Anal. Calcd for $\mathrm{C}_{23} \mathrm{H}_{13} \mathrm{Br}_{2} \mathrm{~N}_{3}$ : C 56.24, H 2.67, N 8.55. Found: C 56.32, H 2.69, N 8.59.

2-Chloro-9-methoxy-11-(5-methoxy-1H-indol-3-yl)-6H-indolo[2,3-b]quinoline (3f): ${ }^{8 \mathrm{~h}}$ Yellow crystals. 
$\mathrm{mp}>300{ }^{\circ} \mathrm{C}$; IR (KBr): v 3343, 3058, 1615, $1595 \mathrm{~cm}^{-1} ;{ }^{1} \mathrm{H}$ NMR (400 MHz, $\left.\mathrm{CDCl}_{3}\right): \delta 3.55$ (s, $\left.3 \mathrm{H}\right), 3.88$ $(\mathrm{s}, 3 \mathrm{H}), 6.01(\mathrm{~s}, 1 \mathrm{H}), 7.88(\mathrm{~d}, J=6.8 \mathrm{~Hz}, 1 \mathrm{H}), 7.22(\mathrm{~d}, J=6.4 \mathrm{~Hz}, 1 \mathrm{H}), 7.45-7.50(\mathrm{~m}, 3 \mathrm{H}), 7.59(\mathrm{~d}, J=$ $8.2 \mathrm{~Hz}, 1 \mathrm{H}), 7.84(\mathrm{~d}, J=8.0 \mathrm{~Hz}, 1 \mathrm{H}), 7.94(\mathrm{~s}, 1 \mathrm{H}), 8.23$ (d, $J=7.4 \mathrm{~Hz}, 1 \mathrm{H}) 10.8(\mathrm{~s}, 1 \mathrm{H}), 11.7(\mathrm{~s}, 1 \mathrm{H}) .{ }^{13} \mathrm{C}$ NMR (100 MHz, $\left.\mathrm{CDCl}_{3}\right): \delta 55.6,56.0,101.2,103.4,107.0,112.7,113.4,119.8,119.9,120.3,121.8$, $124.4,126.5,127.3,127.8,128.2$, 129.2, 131.7, 132.0, 132.8, 139.5, 142.5, 146.0, 153.9, 154.3. Anal. Calcd for $\mathrm{C}_{25} \mathrm{H}_{18} \mathrm{ClN}_{3} \mathrm{O}$ : C 70.18, H 4.24, N 9.82. Found: C 70.24, H 4.27, N 9.85.

11-(5-Cyano-1 $\boldsymbol{H}$-indol-3-yl)-6H-indolo[2,3-b]quinoline-9-carbonitrile (3g): ${ }^{\text {a }}$ Yellow crystals. mp > $300{ }^{\circ} \mathrm{C}$; IR (KBr): v 3352, 3028, 2224, 2209, 1605, $1584 \mathrm{~cm}^{-1} ;{ }^{1} \mathrm{H}$ NMR $\left(400 \mathrm{MHz}, \mathrm{CDCl}_{3}\right): \delta 7.34(\mathrm{~d}, J$ $=8.4 \mathrm{~Hz}, 1 \mathrm{H}), 7.41-7.44(\mathrm{~m}, 2 \mathrm{H}), 7.54(\mathrm{~d}, J=8.0 \mathrm{~Hz}, 1 \mathrm{H}), 7.61-7.63(\mathrm{~m}, 3 \mathrm{H}), 7.79(\mathrm{~s}, 1 \mathrm{H}), 7.98(\mathrm{~s}, 1 \mathrm{H})$, $8.14(\mathrm{~d}, J=8.4 \mathrm{~Hz}, 1 \mathrm{H}), 8.39(\mathrm{~s}, 1 \mathrm{H}), 11.2(\mathrm{~s}, 1 \mathrm{H}), 12.1(\mathrm{~s}, 1 \mathrm{H}) .{ }^{13} \mathrm{C} \mathrm{NMR}\left(100 \mathrm{MHz}, \mathrm{CDCl}_{3}\right): \delta 101.6$, $102.3,108.2$, 113.3, 113.9, 120.4, 120.5, 120.9, 122.1, 125.1, 125.4, 125.6, 126.1, 126.4, 126.7, 127.0, 127.2, 129.8, 130.1, 132.5, 132.9, 138.8, 144.4, 144.9, 146.3. Anal. Calcd for $\mathrm{C}_{25} \mathrm{H}_{13} \mathrm{~N}_{5}$ : C 78.32, H 3.42, N 18.27. Found: C 78.39, H 3.46, N 18.29.

2-Chloro-11-(1H-indol-3-yl)-6H-indolo[2,3-b]quinoline (3h): Yellow crystals. $\mathrm{mp}>300{ }^{\circ} \mathrm{C}$; $\mathrm{IR}(\mathrm{KBr})$ : v 3317, 3046, 1613, $1580 \mathrm{~cm}^{-1} ;{ }^{1} \mathrm{H}$ NMR (400 MHz, $\left.\mathrm{CDCl}_{3}\right): \delta 7.53$ (dd, $\left.J=7.6,8.4 \mathrm{~Hz}, 1 \mathrm{H}\right), 7.58-7.61$ (m, 2H), $7.65(\mathrm{~s}, 1 \mathrm{H}), 7.69$ (dd, $J=7.6,8.0 \mathrm{~Hz}, 1 \mathrm{H}), 7.77(\mathrm{dd}, J=7.6,8.4 \mathrm{~Hz}, 1 \mathrm{H}), 7.87-7.92(\mathrm{~m}, 2 \mathrm{H})$, $7.79(\mathrm{~s}, 1 \mathrm{H}), 8.10(\mathrm{~s}, 1 \mathrm{H}), 8.26(\mathrm{~d}, J=7.8 \mathrm{~Hz}, 1 \mathrm{H}), 8.39$ (s, 1H), 11.49 (s, 1H), $12.39(\mathrm{~s}, 1 \mathrm{H}) .{ }^{13} \mathrm{C} \mathrm{NMR}$ (100 MHz, $\left.\mathrm{CDCl}_{3}\right): \delta$ 106.6, 111.9, 112.9, 114.3, 114.7, 119.4, 121.8, 123.4, 124.0, 124.9, 126.8, 127.0, 128.9, 129.0, 130.0, 131.7, 132.4, 132.7, 135.7, 142.6, 143.2, 144.7, 170.7. Anal. Calcd for $\mathrm{C}_{23} \mathrm{H}_{12} \mathrm{Br}_{2} \mathrm{ClN}_{3}$ : C 52.56, H 2.30, N 7.99. Found: C 52.63, H 2.33, N 8.04.

\section{ACKNOWLEDGEMENTS}

The authors thank Fund of National Science Foundation of China (81274010), Heilongjiang Province Outstanding Youth Fund (JC201101) and innovation team project of Liaoning Province Education Department (Grant No. 2015001) for financial support.

\section{REFERENCES}

1. A. J. Kochanowska-Karamyan and M. T. Hamann, Chem. Rev., 2010, 110, 4489.

2. S. W. Yang, M. Abdel-Kader, S. Malone, M. C. M. Werkhoven, H. W. Jan, I. Bursuker, K. Neddermann, C. Fairchild, C. Raventos-Suarez, A. T. Menendez, K. Lane, and D. G. I. Kingston, J. Nat. Prod., 1999, 62, 976.

3. A. Molina, J. J. Vaquero, J. L. Garcia-Navio, J. Alvarez-Builla, B. Pascula-Terasa, F. Gago, M. M. Rodrigo, and M. Ballestros, J. Org. Chem., 1996, 61, 5587.

4. L. Guittat, P. Alberti, F. Rosu, S. Van Miert, E. Thetiot, L. Pieters, V. Gabelica, D. E. Pauw, A. 
Ottaviani, J. F. Riou, and J. L. Mergny, Biochimie, 2003, 85, 535.

5. G. S. M. Sundaram, C. Venkatesh, U. K. Syam Kumar, H. Ila, and H. Junjappa, J. Org. Chem., 2004, 69, 5760 .

6. $\quad$ K. Cimanga, T. DeBruyne, L. Pieters, and A. J. Vlietinck, J. Nat. Prod., 1997, 60, 688.

7. (a) L. Kaczmarek, R. Balicki, P. Nantka-Namirski, W. Peczynska-Czoch, and M. Mordarski, Arch. Pharm., 1998, 321, 463; (b) W. Peczynska-Czoch, F. Pognan, L. Kaczmarek, and J. Boratynski, J. Med. Chem., 1994, 37, 3503.

8. (a) C. Challa, J. Ravindran, M. M. Konai, S. Varughese, J. Jacob, B. S. D. Kumar, J. Haldar, and R. Lankalapalli, ACS Omega, 2017, 2, 5187; (b) H. K. Kadam, D. D. Malik, L. Salgaonkar, K. Mandrekar, and S. G. Tilve, Synth. Commun., 2017, 47, 1980; (c) L. Fan, M.-L. Liu, Y. Ye, and G.-D.Yin, Org. Lett., 2017, 19, 186; (d) Z. Yan, C. Wan, J. Wan, and Z. Wang, Org. Biomol. Chem., 2016, 14, 4405; (e) H. K. Kadam and S. G. Tilve, J. Heterocycl. Chem., 2016, 53, 2066; (f) A. B. Bracca, D. A. Heredia, E. L. Larghi, and T. S. Kaufman, Eur. J. Org. Chem., 2014, 7979; (g) S. Ali, Y. Li, S. Anwar, F. Yang, Z. Chen, and Y. Liang, J. Org. Chem., 2012, 77, 424; (h) S. Sharma and B. Kundu, Tetrahedron Lett., 2008, 49, 7062; (i) H. K. Kadam, P. T. Parvatkar, and S. G. Tilve, Synthesis, 2012, 44, 1339.

9. (a) F. Tamaddon and A. D. Tafti, Synlett., 2016, 27, 2217; (b) O. S. Nayal, V. Bhatt, S. Sharma, and N. Kumar, J. Org. Chem., 2015, 80, 5912; (c) H. Venkatesan, F. M. Hocutt, T. K. Jones, and M. H. Rabinowitz, J. Org. Chem., 2010, 75, 3488; (d) D. Sawant, R. Kumar, P. R. Maulik, and B. Kundu, Org. Lett., 2006, 8, 1525.

10. (a) L. F. Tietze, G. Brasche, and K. M. Gericke, Domino Reactions in Organic Synthesis; Wiley-VCH: Weinheim, 2006; (b) L. F. Tietze, Chem. Rev., 2006, 96, 115; (c) R. Breinbauer, Synthesis, 2007, 794; (d) S. Indumathi, J. C. Menendez, and S. Perumal, Curr. Org. Chem., 2013, 17, 2038; (e) L. G. Voskressensky, A. A. Festa, and A. V. Varlamov, Tetrahedron, 2014, 70, 551.

11. (a) T. Zhou, D.-L. Wang, J. Xu, L. Yan, and W. Ma, Heterocycles, 2017, 94, 1958; (b) J.-N. Yang, D.-L. Wang, and J.-H. Qian, Heterocycles, 2017, 94, 55; (c) T. Zhou, G.-Y. Pan, D.-L. Wang, and J.-H. Qian, Heterocycles, 2017, 94, 237; (d) T. Zhou, G.-Y. Pan, D.-L. Wang, and J.-H. Qian, Heterocycles, 2017, 94, 957; (e) D.-L. Wang, J. Ma, X.-C. Shi, and J.-Y. Wu, Heterocycles, 2016, 92, 133.

12. (a) J. S. Yadav, B. V. S. Reddy, and S. Sunitha, Adv. Synth. Catal., 2003, 345, 349; (b) C.-J. Liu and C.-J. Yu, J. Heterocycl. Chem., 2011, 48, 845.

13. H.-W. Gong and Z.-f. Xie, Chin. J. Org. Chem., 2012, 32, 1195. 